\title{
A note on antennas: Definitions and methods
}

\section{Bach, Henning}

\section{Published in:}

I E E E Transactions on Antennas and Propagation

Publication date:

1987

\section{Document Version}

Publisher's PDF, also known as Version of record

Link back to DTU Orbit

Citation (APA):

Bach, H. (1987). A note on antennas: Definitions and methods. I E E E Transactions on Antennas and Propagation, 35(3), 325-327.

\section{General rights}

Copyright and moral rights for the publications made accessible in the public portal are retained by the authors and/or other copyright owners and it is a condition of accessing publications that users recognise and abide by the legal requirements associated with these rights.

- Users may download and print one copy of any publication from the public portal for the purpose of private study or research.

- You may not further distribute the material or use it for any profit-making activity or commercial gain

- You may freely distribute the URL identifying the publication in the public portal

If you believe that this document breaches copyright please contact us providing details, and we will remove access to the work immediately and investigate your claim 


\section{Communications}

\section{A Note on Antennas: Definitions and Methods}

\section{HENNING BACH}

\begin{abstract}
Definitions of scattered and diffracted fields, originally given by R. F. Millar, are reviewed and supplemented. The definitions are used to discuss relations between results obtained by commonly used pattern prediction methods for reflector antennas.
\end{abstract}

The concepts of scattering and diffraction are essential in most applications of maxwellian theory. As an example we may mention the synthesis, analysis, and design of antennas, in particular reflector antennas, where various methods are used to determine the scattered or diffracted fields, which are produced by the reflectors. It is the purpose of this communication to discuss the definition of these concepts and in particular to classify some of the methods used for their computation.

In 1965 R. F. Millar addressed the questions about definitions in some lecture notes on scattering and diffraction [1]. We here quote, with the omission of a few statements, the following discussion from the notes:

"We yet have to give a precise definition of what we mean by 'diffraction' and 'scattering.' To some extent, the choice of terminology is a matter of personal preference; however, it is felt that the following discussion is in agreement with general usage.

"Suppose that a source in a homogeneous medium emits waves which may be described by a function of position and time $U_{i}$. When some form of obstacle is introduced into this field, the total field is modified and is not described by the function $U_{i}$ alone. This change is attributed to 'diffraction' or 'scattering,' depending on the circumstances. Each term refers to a different possible description of the same total field. In some problems one term is more appropriate than the other, but in many other cases both are used indiscriminately.

"After introduction of the obstacle, let the total field be $U$, and define the 'scattered field' $U_{s}$ by $U=U_{i}+U_{s}$, where $U_{i}$ is the free space field. It may be possible also to define a 'diffracted field' $U_{d}$ by $U=U_{g}+U_{d}$; here, $U_{g}$ denotes the field of 'geometrical optics,' obtained by the consideration of reflected and refracted rays. Because $U_{s}$ is always defined, it is always possible to speak of 'scattering.' In cases where there is a well-defined geometrical optics solution $U_{g}$, and $U_{d} \neq 0$, we may speak of 'diffraction' or 'scattering.' If $U_{g}$ is not welldefined, or if $U_{d}=0$, then it is customary to speak of 'scattering.' Thus, we see that the term 'scattering' refers to any change produced in the total field by a scatterer, while 'diffraction' is related to the existence of a geometrical optics field $U_{g}$. There is scattering without diffraction when $U=U_{g}$ $\neq U_{i}$. We speak of diffraction by an aperture in a plane

Manuscript received December 26, 1985; revised March 11, 1986.

The author is with the Electromagnetics Institute, Technical University of Denmark, DR-2800, Lyngby, Denmark.

IEEE Log Number 8621491. because the 'removal' of the aperture leads to the vanishing of $U_{d}$ and there is no diffraction. But one does not refer to scattering by an aperture because the absence of the aperture does not imply the vanishing of the scattered field.

"It is believed that these interpretations of the terms 'scattering' and 'diffraction' are generally accepted. However, as mentioned previously, the choice of terminology is to some extent a personal matter."

It is noted that Millar expresses his definitions, which in this communication are applied to time harmonic, steady state fields, through equations, which makes them clear and unambiguous. Furthermore, it seems still to hold true that his definitions are generally accepted by a majority of antenna researchers.

In the following we shall use Millar's definitions to discuss methods for the computation of the field from reflector antennas. To account for the problem of mutual interactions between the primary source and the reflector, we shall supplement Millar's definition of the incident field by the assumption that the field originates from a primary source defined by a distribution of impressed currents. Furthermore, for simplicity and since this will not limit the generality of our discussion, we shall consider only a single reflector antenna with a uniformly illuminated aperture, which is large in terms of wavelengths, such that the geometrical optics field solution is well defined.

The structure of the field around a reflector antenna usually is different in various regions. As examples of such regions we may mention the main lobe, the side lobe and the back lobe regions, which pertain to field pattern characteristics. Depending upon the Rayleigh distance we speak of near field, Fraunhofer, far field and Fresnel zones, while terms like shadow and reflection boundaries, diffraction zones, interference zones, focal and caustic regions are related to geometrical optics. These structural differences often imply that more than one method of computation or a combination of such methods must be used in order to calculate the entire field around the antenna.

The methods in common use may be classified in three main groups, namely:

- current integration methods

- aperture integral methods

- quasi-optical methods.

The oldest and probably most widely used methods are the current integration methods, which are based upon direct computation of the fields radiated by the currents induced on the reflector by the primary source. If the exact currents on the reflector, back as well as front, are known, the exact field from the antenna may be found simply by calculating a surface integral.

In principle, the exact current distribution may be found using integral equation methods [2]. In practice the integral equations are solved numerically, e.g., by moment methods techniques [3], and solutions, which ideally approach exact solutions to Maxwell's equations, are obtained. Despite these facts the integral equation techniques are not widely used due to excessive computer time requirements even for small reflector antennas. However, such numerical solutions may serve as useful reference solutions for comparisons of results obtained by other methods [4]. Since in most 
cases the current distribution cannot be determined exactly, an approximated current distribution must normally be introduced. The usual approximation is the physical optics approximation, where the true current distribution is substituted by a truncated distribution, $\bar{J}_{\text {po }}$, found using the well-known formula $\bar{J}_{p o}=2 \hat{n} \times H_{i}$, which assumes that the incident field reflects as if the surface of the reflector at each point were part of an infinite perfectly conducting plane. It appears that the physical optics field is essentially the field from a finite, twodimensional continuous array of infinitesimal elements and that the lobe structure of the physical optics field pattern is therefore identical in nature to that of array patterns. To obtain the total field an additional term must be added. We shall refer to this term as the fringe wave field which we, in analog to Millar's above definitions, define as the field $U_{\mathrm{fw}}$ which must be added to the incident field $U_{i}$ and the physical optics field $U_{\mathrm{po}}$ to obtain the total field $U$, i.e., $U=$ $U_{i}+U_{\mathrm{po}}+U_{\mathrm{fw}}$.

The aperture integral methods are based on the field equivalence principle [5]. Thus if the exact tangential electric or magnetic field is known on a closed surface enclosing the antenna, the exact field from the antenna may be found simply by calculating a surface integral. Recently it has been shown analytically that if the aperture surface caps the reflector, then the fields determined by integration of the exact current on the illuminated side of the reflector are identical to the fields determined by an aperture integration of the exact tangential fields [7].

The aperture integral methods exist in several versions characterized by the shape of the closed surface and the technique used to compute the outside field. The classical approach is well known, where the surface is taken as the aperture plane of the reflector plus its backside [5]. The usual approximation in this case is the geometrical optics approximation since the aperture field is computed using geometrical optics. The radiation field then, neglecting the contribution from the backside current, may be obtained for example using fast Fourier transform (FFT) technique. Thus the classical integral method neglects the edge effect and is in this respect similar to the physical optics technique. In [7] it is pointed out that both methods rely on the locally plane wave approximation and therefore, within this approximation, yield equal co-polar far fields. As an example of an extended aperture method we mention the spherical near-field geometrical theory of diffraction (SNFGTD) method [6], where the closed surface is a sphere surrounding the antenna and spherical wave expansions are used to obtain the far field. Also other extended aperture methods, where the closed surface includes points at infinity, are in use.

The last group of methods comprises the classical geometrical optics supplemented by the recently developed quasi-optical methods. As in the above cases, if the diffracted field using Millar's definition is defined at all and can be found, the exact field produced by the antenna may be found also by these methods. In caustic regions, as for example the main beam region of a collimated antenna, the ray optical field description is not valid. Thus the quasioptical methods cannot be applied and so called caustic corrections must be used in such regions. Furthermore the methods are asymptotic in nature, for which reason they are strictly valid only for zero wavelengths.

Now let us introduce the following notation:

$E$ total field from the antenna

$E_{s} \quad$ scattered field according to Millar's definition

$E_{\text {po }} \quad$ physical optics field

$E_{\mathrm{fw}}$ fringe wave field

$E_{i} \quad$ free space field from the primary source

$E_{r} \quad$ reflected field

$E_{d} \quad$ direct field from the primary source

$E_{\mathrm{go}} \quad$ geometrical optics field $=E_{d}+E_{r}$

\section{$E_{\text {dif }}$ diffracted field according to Millar's definition.}

Using these symbols we may write

$$
E=E_{i}+E_{s}=E_{i}+E_{\mathrm{po}}+E_{\mathrm{fw}}=E_{\mathrm{go}}+E_{\mathrm{dif}}
$$

which merely expresses the fact that the field from the antenna may be described in several different ways. With these equations at hand we may now discuss the relations between the various field constitutents.

For example, we may derive the relation

$$
E_{s}=E_{\mathrm{po}}+E_{\mathrm{fw}} \text {. }
$$

The equation shows that $E_{\mathrm{po}}$ is a good approximation to the scattered field provided $E_{\mathrm{fw}} \ll E_{\mathrm{po}}$. Since, as pointed out above, the physical optics field is essentially an array field $E_{\mathrm{po}}$ must be largest where all elements are in phase, i.e., the main beam direction. Thus we may expect physical optics to be very close to the scattered field in and close to the main beam direction. This fact is the main justification for the widespread use of physical optics. In other directions, for example in the sidelobe regions, where the fields from most array elements interfere destructively, only array elements close to the rim of the reflector having no neighboring elements contribute significantly to the resulting physical optics field. Thus in such directions the fringe wave field cannot be neglected.

In order to discuss the fields obtained by the quasioptical methods we have to consider separately the interference zone 1 , the diffraction zone 3 and the intermediate zone 2, shown in Fig. 1. In the three zones we find

$$
E_{\mathrm{go}}=\left\{\begin{array}{l}
E_{d}+E_{r}=E_{i}+E_{r} \\
E_{d}=E_{i} \\
0=0 .
\end{array}\right.
$$

In cases where collimation of the geometrical optics field is present, the above equations may become singular. This case is discussed in detail below. Assuming for the moment that no such caustics are present the scattered field is given by

$$
E_{s}=\left\{\begin{array}{l}
E_{r}+E_{\mathrm{dif}} \\
E_{\mathrm{dif}} \\
-E_{i}+E_{\mathrm{dif}}
\end{array}\right.
$$

and we obtain the following relations:

$$
E_{\mathrm{po}}=\left\{\begin{array}{l}
E_{\mathrm{r}}+E_{\mathrm{dif}}-E_{\mathrm{fw}} \\
E_{\mathrm{dif}}-E_{\mathrm{fw}} \\
-E_{i}+E_{\mathrm{dif}}-E_{\mathrm{fw}} .
\end{array}\right.
$$

From these equations it is learned that the field computed by physical optics includes some diffraction constitutents, unless $E_{\mathrm{dif}}=E_{\mathrm{fw}}$ which is probably never the case. On the other hand, using Millar's definitions, the physical optics field never includes such diffraction constitutents that are not included in the diffracted field.

In cases where the field in the interference region 1 is collimated the geometrical optics description breaks down and the field must be found by other methods, usually physical optics. Thus, neglecting $E_{i}$ and $E_{\mathrm{fw}}$, we arrive at the pattern prediction scheme

$$
E_{s}= \begin{cases}E_{\mathrm{po}}, & \text { in the caustic region } \\ E_{\mathrm{go}}+E_{\mathrm{dif}}, & \text { outside the caustic region }\end{cases}
$$

This probably is the most widely used technique of all.

The crucial question now remains: how are the field constitutents discussed above calculated in practice? We already addressed this question for the aperture integral methods and for the integral 


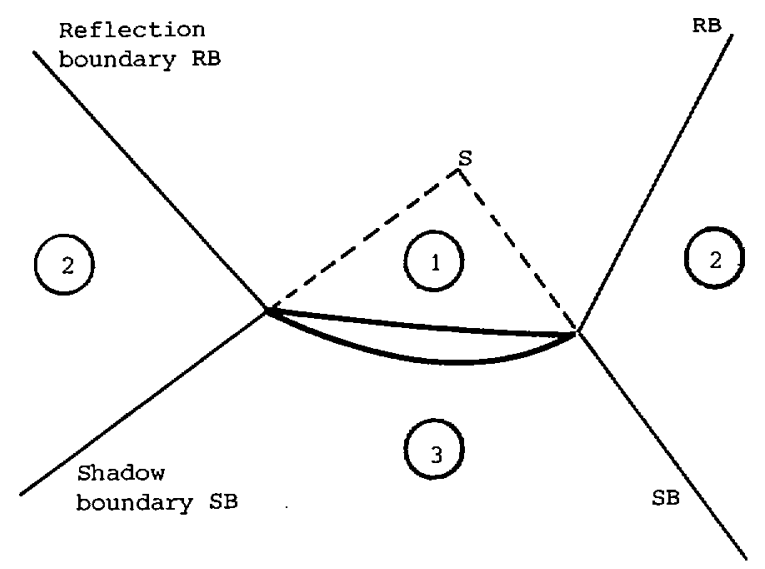

Fig. 1. The geometrical optics regions around a scatterer illuminated by a primary source $S$. Interference zone 1 , intermediate zone 2 , and diffraction zone 3.

equation technique for the determination of the surface currents. With regard to computation of the surface integrals, including the physical optics integral, special numerical techniques have been developed which allow a fast and accurate evaluation of the integral even for very large reflectors. With regard to the fringe wave field and the diffracted fields, the physical theory of diffraction and the geometrical theory of diffraction, respectively, offers very good approximations to these fields. Thus all of the above mentioned methods, when used correctly and to the limit of their accuracy for the prediction of patterns of reflector antennas, yield co-polar patterns which are practically identical, i.e., even with the best measurement techmique available at hand, it is not in general possible to prefer one method to another.

However, when computing very low near-in sidelobes or weak cross-polar fields which are important tasks in many applications as for example satellite communication antennas, the various theories yield results which may differ considerably. It is very probable that the success of further research in this area, which is certainly necessary in order to solve the problems, is dependent on clear definitions of the concepts involved, and a thorough understanding of the nature of the methods used.

\section{ACKNOWLEDGMENT}

The author is indebted to Dr. Arthur Yaghjian for valuable discussions and suggestions which contributed to the work reported.

\section{REFERENCES}

[1] R. F. Millar, "Diffraction and scattering of waves," Electromagn. Inst., Tech. Univ. Denmark, NB 15, 1965.

[2] D. S. Jones, Methods in Electromagnetic Wave Propagation. London, U.K.: Oxford Univ. Press, 1979.

[3] R. F. Harrington, Field Computations by Moment Methods. New York: Macmillan, 1968.

[4] H. Bach, "Comments on 'A new method of analysis of the near and far fields of parabolic reflectors'," IEEE Trans. Antennas Propagat., vol. AP-33, no. 1, pp. 116-117, Jan. 1985.

[5] R. E. Collin and F. J. Zucker, Antenna Theory. New York: McGraw-Hill, 1969, pp. 69-74.

[6] H. Bach, A. Frandsen, and F. H. Larsen, "Pattern prediction for a focused reflector antenna using GTD and near field to far field transformations," presented at 2nd Int. Conf. Antennas Propagat. York, England, 1981.

[7] A. D. Yaghjian, "Equivalence of surface current and aperture field integrations for reflector antennas," IEEE Trans. Antennas Propagat., vol. AP-32, no. 12, pp. 1355-1358, Dec. 1984.

\section{The Coaxial Collinear Antenna: Current Distribution from the Cylindrical Antenna Equation}

\author{
THIERRY J. JUDASZ, WARNER L. ECKLUND, AND BEN B. BALSLEY
}

\begin{abstract}
A theoretical model and computer simulations of the current on the coaxial collinear (COCO) antenna are presented. Comparisons between numerical results and measurements are made for 24- and 26-element COCO antennas at $50 \mathrm{MHz}$ and for six- and eight-element CoCO anteninas at $915 \mathrm{MHz}$. The results show reasonable agreement between first-order theory and measurements.
\end{abstract}

\section{INTRODUCTION}

The coaxial collinear antenna or COCO antenna as described by Balsley and Ecklund [1] has been used successfully in a number of radars and communication antennas for many years. The mesosphere-stratosphere-troposphere (MST) radar at Poker Flat, AK, is made of 256 separate coaxial collinear antennas constructed from coaxial cable [2]. The Jicamarca radar observatory in Peru, on the other hand, incorporates a large array containing 1536 separate coaxial collinear antennas constructed using aluminum tubing [3]. The main advantage of such arrays is that an array of $n n$-element coaxial collinear antennas is made of $n^{2}$ dipoles and has $n$ feed points, whereas a $n \times n$ dipole array is made of $n^{2}$ dipoles also, but has $n^{2}$ feed points. Some commercial communication antennas are made of vertical COCO antennas and are used in the range $400-520 \mathrm{MHz}$. In spite of its relatively wide usage, however, a thorough theoretical description of the COCO antenna has not been attempted. We present here a short note outlining a first-order theoretical model which explains the general behavior of the COCO antenna.

\section{DESCRIPTION OF THE MODEL}

The COCO antenna consists of pieces of coaxial line connected together so that a wave propagates inside the line and another propagates outside. The elements are $\lambda c / 2$ long, where $\lambda c$ is the wavelength in the cable. The transposition of the inner and outer conductors (see Fig. 1) forces the phase of the current on the outer conductor to remain roughly constant, in contrast to the periodic $180^{\circ}$ phase reversal on the outer conductor of a long cylindrical antenna. The overall length of the antenna of radius $a$ is $2 h$. We define $\lambda$ to be the wavelength of the free-space wave, and assume $a / \lambda \ll 1$. $k$ is the free-space propagation constant. The antenna is fed at the center connection only.

If the elements of the antenna are $\lambda c / 2$ long, if the inner-to-outer conductor connections are of negligible length, and if the central source is a delta function generator of voltage $V$, then as a first approximation, the electric field component parallel to the antenna axis about the center connection can be expressed as

$$
E_{z}(z)=V \delta(z),
$$

and, from transmission line theory, the voltage generators at the connections $\left(z_{1}, z_{2}, \cdots, z_{n}\right)$ of the COCO antenna (see Fig. 1) can be expressed approximately as $V \delta\left(z-z_{i}\right)$, because in (2) below $\sin \left(k_{c} x\right)=0$ and $\cos \left(k_{c} x\right)=-1$ when $x=\lambda c / 2$. Hence the electric field component parallel to the antenna axis about the connection $z_{i}$ can be expressed as $E_{z}(z)=-(-V) \delta\left(z-z_{i}\right)=V \delta\left(z-z_{i}\right)$,

Manuscript received May 25, 1986; revised September 16, 1986.

T. J. Judasz is with the Cooperative Institute for Research in Environmental Sciences, University of Colorado, Boulder, CO 80309.

W. L. Ecklund and B. B. Balsley are with the Aeronomy Laboratory, National Oceanic and Atmospheric Administration, Boulder, CO 80303.

IEEE Log Number 8612500. 